\title{
Effect of mixing conditions on the tensile properties of ethylene vinyl acetate/waste tire dust (EVA/WTD) blend
}

\begin{abstract}
Blends of Ethylene Vinyl Acetate/Waste Tire Dust (EVA/WTD) were prepared by using a Haake Rheomix at 100/0, 90/10, 80/20, 70/30, and 60/40 blend ratios. The effect of mixing temperature, blend ratio and blending time on the tensile properties of EVA/WTD blends were investigated. The mixing time was varied from 5 to 30 minutes, while the mixing temperature was varied from 120 to $160^{\circ} \mathrm{C}$. The tensile properties of the blends found to show a gradual decrease with the addition of WTD. EVA/WTD blends prepared at $140^{\circ} \mathrm{C}$ mixing temperature and $10 \mathrm{~min}$ mixing time found to be suitable mixing parameters to obtain optimum blend properties. In general, declines in the EVA/WTD blends properties were also observed with increase in mixing time and temperature.
\end{abstract}

Keyword: Ethylene vinyl acetate; Mixing condition; Waste tire dust 